\title{
Resonant spectroscopy of electric-field-induced superlattices
}

\author{
S. A. Vitusevich, ${ }^{\text {a) }}$ A. Förster, and H. Lüth \\ Institut für Schichten und Grenzflächen (ISG), Forschungszentrum Jülich, D-52425 Jülich, Germany \\ A. E. Belyaev, S. V. Danylyuk, and R. V. Konakova \\ Institute of Semiconductor Physics, National Academy of Sciences of Ukraine, 03028 Kiev, Ukraine \\ D. I. Sheka \\ Radiophysics Faculty, National Taras Shevchenko University of Kiev, 03127 Kiev, Ukraine
}

(Received 12 January 2001; accepted for publication 14 June 2001)

\begin{abstract}
A system of interdigital gates is used to create a periodic potential profile in a multilayer heterostructure. The electrostatic problem for the spatial distribution of the potential is solved and experimentally examined by measurements of current-voltage characteristics of resonant-tunnelling diodes embedded in the depletion region of the Schottky contact. It is shown that the position of the resonant peak voltage is sensitive to the spatial potential distribution and that with appropriate parameters of the heterostructure the sensitivity of the gates can be considerably enhanced. (C) 2001 American Institute of Physics. [DOI: 10.1063/1.1392956]
\end{abstract}

\section{INTRODUCTION}

In the past few years the use of gates with a specific design has attracted a wide interest in the study of one- and zero-dimensional electron systems. Electrically biased gates allow lateral potentials to be precisely controlled and, as a result, the potential profile of the structures can be deliberately changed. It has been previously demonstrated that a particularly fruitful method of investigating one-dimensional phenomena in electronic transport is the "electrostatic squeezing" of a 2DEG in the heterojunction. This squeezing is produced by a split gate configuration that provides a progressive narrowing of the width of the $2 \mathrm{DEG}{ }^{1,2}$ The formation of a semiconductor dot in an inverted GaAs/AlGaAs heterostructure has also been reported in which electrostatic gates were used to confine and adjust the density of a twodimensional electron gas. ${ }^{3}$ A direct manifestation of the quantum confinement of electrons in a three-terminal resonant-tunnelling diode in which the effective lateral dimension may be varied using a gate electrode has been demonstrated in Ref. 4. A system of interdigitated gates produced on heterostructure surfaces has been successfully employed both in applications (for instance, in improving infrared photodetector parameters ${ }^{5}$ ) and in fundamental studies (for instance, in a comprehensive study of a quantum wire array produced by controllable lateral potentials ${ }^{6}$ ). A possibility for a precise control of the potential amplitude as well as of the lateral potential distribution is interesting both from the perspective of fundamental physics, and also because of the potential for device applications, particularly in the development of a new kind of switcher. Despite this, surprisingly little is known about the detailed profile of the electrostatic potential. Moreover, in all previous papers the appearance of a periodic potential distribution has been discussed qualitatively.

a) On leave from the Institute of Semiconductor Physics, NASU, $03028 \mathrm{Kiev}$, Ukraine.
Here we present a theoretical investigation of the factors influencing the shape and magnitude of the periodic potential distribution arising in realistic heterostructures. We solve an electrostatic problem in an analytical form that allows a calculation of the spatial distribution of the potential and gives reasonable constraints on the permitted models of potential superlattices. The numerical solution with actual device parameters is in good agreement with the experimental data.

\section{EXPERIMENT}

The experimental verification for the formation of a periodic potential profile was performed with the currentvoltage characteristics of a resonant-tunnelling diode (RTD) embedded in a depletion region of a Schottky contact. A top view of the device is given in Fig. 1. The upper electrodes were placed on the surface of the heterostructure in the form of two interdigital gates. Each fingergate consists of $20 \mathrm{Ti}$ strips which are $20 \mu \mathrm{m}$ long and $b \mu \mathrm{m}$ wide $(b=0.5,0.7,1$ $\mu \mathrm{m})$. The distance between the gates, $a$, was varied from 0.5 to $1 \mu \mathrm{m}$. The electrodes represent the Schottky contacts to the multilayer heterostructure with the barrier height $\phi_{0}$. The heterostructure used for the measurements was grown by molecular-beam epitaxy (MBE) on (100) $n^{+}$-GaAs substrate. Growth was initiated with $200 \mathrm{~nm} n^{+}$-GaAs $(n=1$ $\times 10^{18} \mathrm{~cm}^{3}$ ) and $100 \mathrm{~nm}$ undoped GaAs spacer layers followed by $2 \mathrm{~nm} \mathrm{AlAs}-4 \mathrm{~nm}$ GaAs-2 nm AlAs double barrier structure. An undoped GaAs layer serving as a capping layer was varied from 40 to $100 \mathrm{~nm}$ width in different devices. Current-voltage characteristics were measured with an HP4145B semiconductor parameter analyzer operated in transistor mode (common-drain configuration). All measurements were conducted at room temperature.

\section{ELECTROSTATIC PROFILE MODELLING}

Let us assume that one upper electrode is grounded ( $U$ $=0)$ while the second one serves as a gate $\left(U=U_{g}\right)$. Both electrodes are located in the plane $y=h$ (Fig. 1). The doublebarrier resonant-tunnelling structure is placed in the plane $y$ 


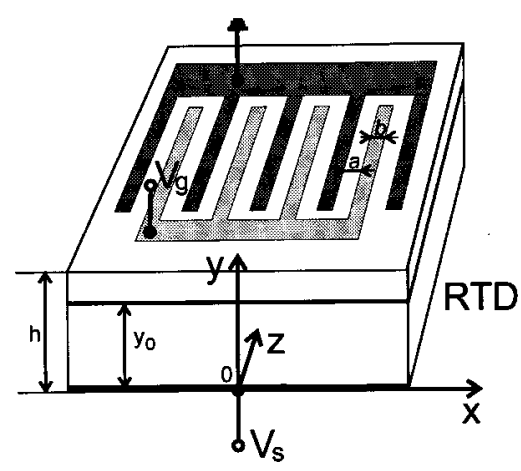

FIG. 1. Top view of the experimental device.

$=y_{0}$ and is used as a spectroscopic probe for potential distribution. The width of the RTD is assumed to be small with respect to the total width of the heterostructure, $h$. The energy of the ground quasibound state of the quantum well (QW) is determined by parameters of the RTD, but being calculated from the QW bottom [i.e., from the potential $U(x, y)$ in the plane $\left.y=y_{0}\right]$ it should be very sensitive to the potential distribution. In Fig. 2 the potential profile of the heterostructure along the growth direction is schematically depicted. If the bottom electrode (substrate) is biased by an external voltage $V_{s}$, its potential relative to the ground will
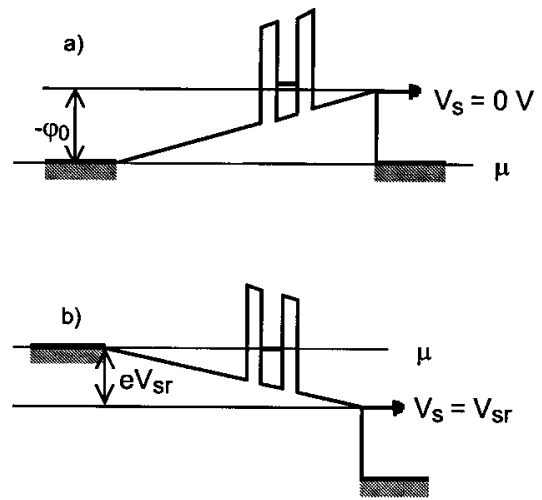

FIG. 2. Schematic cross-sectional diagram of the potential profile of the heterostructure along the growth direction ( $\mu$ and $\phi_{0}$ are emitter Fermi energy and the Schottky barrier height, respectively) at different voltages applied to the substrate, $V_{s}$ : (a) $V_{s}=0 \mathrm{~V}$, (b) $V_{s}=V_{s r}$. For the sake of simplicity we assume a linear voltage drop throughout the structure.

be $U_{s}=e V_{s}+\phi_{0}$. The voltage applied between two upper electrodes (which corresponds to the gate electrode being charged with the potential $U_{g}$ ) leads to the formation of a spatially nonuniform potential distribution in the plane $(x, y)$. In this case the spatial distribution of the potential $U(x, y)$ will be derived by a solution of the Laplace equation

$$
\Delta U(x, y)=0
$$

with the boundary conditions

$$
\begin{aligned}
& U(x, 0)=U_{s}, \\
& U(x, h)= \begin{cases}U_{g} & \text { for }-0.5 b+2 n(a+b) \leqslant x \leqslant 0.5 b+2 n(a+b), \\
0 & \text { for }-0.5 b+(2 n+1)(a+b) \leqslant x \leqslant 0.5 b+(2 n+1)(a+b),\end{cases}
\end{aligned}
$$

where $n=0, \pm 1, \pm 2, \ldots, \pm N$, and the total amount of strips equals $2(2 N+1)$. We can use this procedure because the undoped semiconductor layer fills the space between the electrodes.

The solution of Eqs. (1)-(3) can be found by using the results described in the Appendix where the solution of the auxiliary problem connected with searching the twodimensional distribution of $U_{a}\left(x, y ; v_{0}, v_{h} ; a\right)$ for the area confined by the plane $y=0$ with potential $v_{0}$ and by two semi-infinite planes $y=h, x \geqslant+a / 2$ and $y=h, x \leqslant-a / 2$, whose potentials are $-v_{h}$ and $+v_{h}$, respectively, is given. The result can be represented as follows:

$$
\begin{aligned}
& U\left(x, y ; U_{s}, U_{g} ; b ; a\right) \\
& =\sum_{n=-N}^{N}\left\{U _ { a } \left[x-\left(2 n-\frac{1}{2}\right)(a+b), y ; \frac{v_{0}}{2(2 N+1)},\right.\right. \\
& \left.\quad-\frac{1}{2} v_{h} ; a\right]+U_{a}\left[x-\left(2 n+\frac{1}{2}\right)(a+b), y ; \frac{v_{0}}{2(2 N+1)},\right. \\
& \left.\left.\quad+\frac{1}{2} v_{h} ; a\right]\right\} .
\end{aligned}
$$

Spatial distribution of the potential calculated using Eq. (4) is shown in Fig. 3(a). The following parameters of the structure were used: $a=b=0.53 \mu \mathrm{m}, \quad h=0.148 \mu \mathrm{m}, \quad y_{0}$ $=0.108 \mu \mathrm{m}, V_{g}=5 \mathrm{~V}, V_{s}=-2 \mathrm{~V}$. The potential profile at $y=y_{0}$ is depicted by the bold curve. Figure 3(b) shows the cross sections of the calculated surface for different distances from the top electrodes. The formation of a potential superlattice is clearly seen.

\section{RESULTS AND DISCUSSION}

Figure 4 shows the current-voltage curve of the device at a negatively biased substrate and $V_{g}=0$. A resonance peak in the current-voltage characteristics at around $1 \mathrm{~V}$ is clearly observable. The resonance occurs when the energies of the injected electrons from the emitter align with the lowest electron level in the QW. In our case, the energy of this level $E_{r}$ is calculated to be $183 \mathrm{meV}$. The electron states inside the QW are two dimensional. If $V_{g} \neq 0$, the spatial potential distribution beneath the fingergates becomes modulated as described above. Thus, a lateral confinement arises and at a certain gate voltage a renormalization of the effective con- 

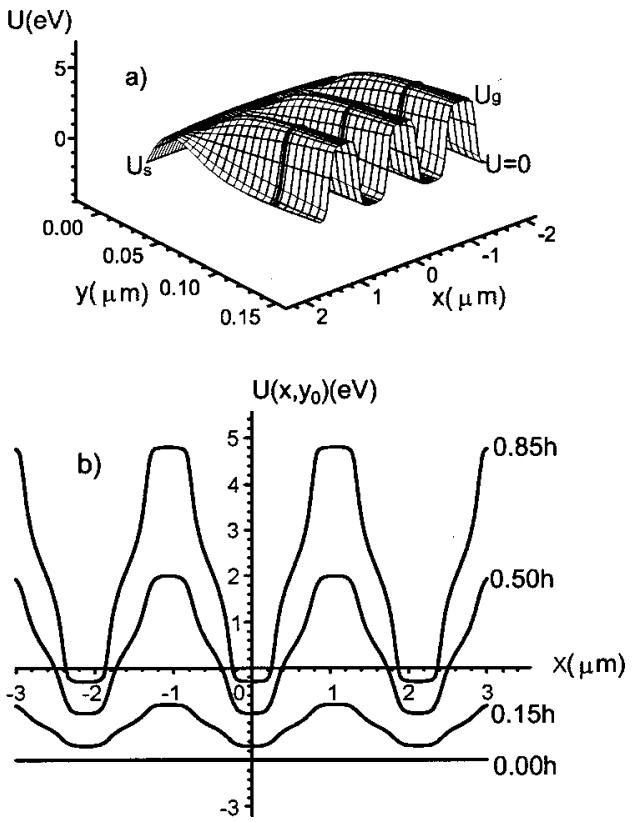

FIG. 3. (a) Spatial potential distribution calculated using Eq. (4). The bold curve corresponds to the position of the electrostatic probe: $V_{g}=5 \mathrm{~V}, V_{s}$ $=-2 \mathrm{~V}$ (b) Cross sections of the calculated surface for different distances from the bottom electrode.

finement potential can transform a two-dimensional QW into a one-dimensional quantum wire array. Simultaneously the spatial potential distribution will block the lateral current. Thus, we can consider the current measured as an integral over the various laterally confined RTDs. We can expect that the position of the resonant peak should be very sensitive to these transforms. Actually, the voltage at which the resonance occurs can be found from

$$
e V_{s r}=-\left[\phi_{0}+U\left(0, y_{0}, U_{s}, U_{g}, a, b\right)+E_{r}\right],
$$

where $U\left(0, y_{0}, U_{s}, U_{g}, a, b\right)$ is the solution of Eq. (4) in the point of the heterostructure with coordinates $\left(0, y_{0}\right)$, i.e., in the middle of the QW just below the center of the grounded electrode. An approximation of the numerical solution of Eq. (5) should be given as follows:

$$
e V_{s r}=-\left(\phi_{0}+0.2508\right)+e V_{g} \exp \left(-12.5 a^{0.77}\right) .
$$

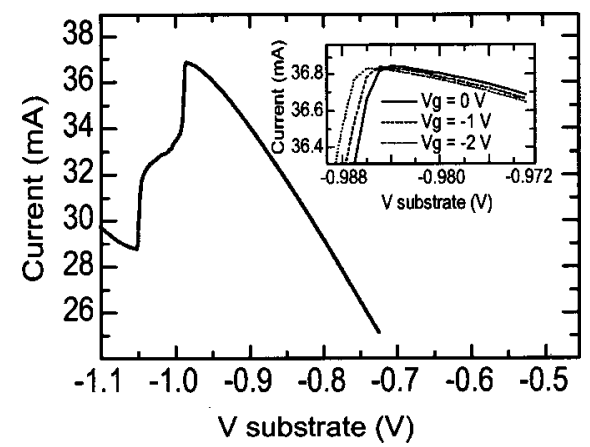

FIG. 4. Current-voltage curve of the device at negatively biased substrate and $V_{g}=0$. Inset: Enlarged picture of the $I-V$ curve at different gate voltages taken in the proximity of the resonance peak.

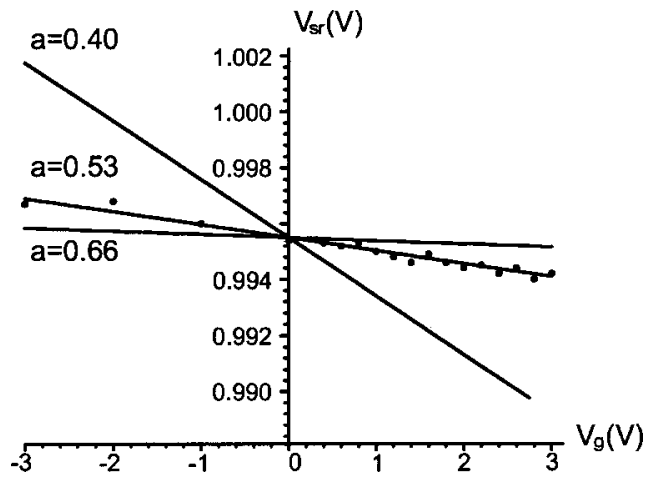

FIG. 5. Dependences of $V_{s r}$ versus $V_{g}$ calculated for different spacing between gates. Circles represent experimental data.

Here we used the following data: $h=0.148 \mu \mathrm{m}, y_{0}$ $=0.108 \mu \mathrm{m}, E_{r}=183 \mathrm{meV}$. The values of $a, b$, and $V_{g}$ were varied: $-3 \mathrm{~V} \leqslant V_{g} \leqslant 3 \mathrm{~V}, 0.05 \mu \mathrm{m} \leqslant a, b \leqslant 1 \mu \mathrm{m}$.

In Fig. 5 we present the results of the calculation of $V_{s r}$ performed for $\phi_{0}=0.7447 \mathrm{eV}$ and $a=b=0.53 \mu \mathrm{m}$. Experimental data (circles in Fig. 5) is taken from the $I-V$ curves measured at different gate voltages (see inset in Fig. 4) fairly consistent with the potential profile modelling. It can be seen from Fig. 5 that for this particular device the resonance peak voltage $V_{s r}$ manifests too weak a dependence on $V_{g}$. Note, however, that the effect is very sensitive with respect to a splitting between the upper electrodes and a deviation of this value of just $20 \%$ leads to a huge change. For comparison, in Fig. 5 purpose we plotted two dependences $V_{s r}=f\left(V_{g}\right)$ calculated for two different values of $a(0.4 \mu \mathrm{m}$ and $0.66 \mu \mathrm{m})$ and assuming the other parameters are unchanged. The results show that appropriate parameters of the heterostructure and gate design can lead to a considerable effect. Indeed, looking at Fig. 6(a) we can see a considerable amplification of the effect if one takes spacing between the gates which is three, five or ten times less than the experimental device. The shape and the amplitude of the potential modulation also undergoes visible changes under these manipulations. They are clearly seen in Fig. 6(b) where the potential profile $U\left(x, y_{0} ; U_{s}, U_{g}\right.$; (a) (b) is plotted as a function of the normalized coordinate $x / a$. There are two important consequences that follow from the above-mentioned simulation. First, if we wish to obtain a strong dependence $V_{s r}$ $=f\left(V_{g}\right)$, we have to decrease the spacing between the gates. The situation is very promising for the development of a new kind of electronic switcher. The second consequence consists in the possibility of controlling a lateral potential confinement. To exploit this possibility we should place the electrostatic probe (in our case a double-barrier structure) closer to the fingergates. This could introduce new aspects of the physics of potential superlattices.

\section{CONCLUSIONS}

We used the system of interlocked gates fabricated on the surface of a heterostructure to produce a periodic potential distribution in its depth. The theoretical analysis allowed an investigation of different factors influencing the shape and the magnitude of the periodic potential distribution. A 

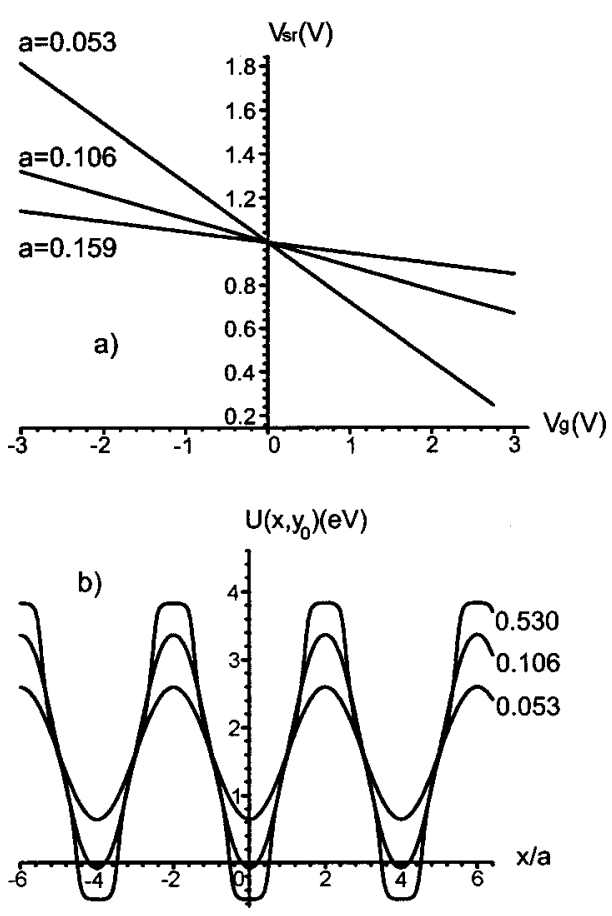

FIG. 6. (a) Dependences of $V_{s r}$ versus $V_{g}$ calculated for spacing between gates equal to $53 \mathrm{~nm}, 106 \mathrm{~nm}$, and $159 \mathrm{~nm}$. (b) Lateral potential distribution calculated for different spacing between gates as a function of the reduced coordinate $x / a$ and taken at the position of the electrostatic probe.

double-barrier resonant-tunnelling structure placed in the depletion region of a Schottky contact was used as a spectroscopic probe for a potential distribution. The shift of the resonance peak of the $I-V$ curve observed in the experiment was fairly consistent with the potential profile modelling. Our results demonstrate that with appropriate parameters of the heterostructure and gate design the effect could be enhanced considerably. This could be used in a new kind of electronic switcher based on the resonance tunnelling effect.

\section{ACKNOWLEDGMENTS}

Financial support by the Deutsche Forschungsgemeinschaft (A.E.B) and Alexander von Humbolt Foundation (S.A.V) is gratefully acknowledged.

\section{APPENDIX: ELECTROSTATIC POTENTIAL IN A PLANE-PARALLEL THREE-GATE SYSTEM}

Let us determine the potential distribution $U_{a}\left(x, y ; v_{0}, v_{h} ; a\right)$ in the space confined by the plane $y=0$ with the potential $v_{0}$, and by two semi-infinite planes $y$ $=h, x \geqslant a / 2$ and $y=h, x \leqslant-a / 2$, whose potentials are $-v_{h}$ and $+v_{h}$, respectively. We consider the potential to be determined in the plane by a complex variable $z=x+i y$ and perform the conform transformation to the variable $w_{1}=u_{1}$ $+i v_{1}$ in the following manner: ${ }^{7}$

$$
z=h\left(w_{1}+A \cdot \tanh \frac{\pi w_{1}}{2}\right),
$$

where $A$ is a constant that to be determined. With respect to the variables $x$ and $y$ the transform (A1) has the form

$$
\begin{aligned}
& x=h\left(u_{1}+A \cdot \frac{\sinh \pi u_{1}}{\cosh \pi u_{1}+\cos \pi v_{1}}\right), \\
& y=h\left(v_{1}+A \cdot \frac{\sin \pi v_{1}}{\cosh \pi u_{1}+\cos \pi v_{1}}\right) .
\end{aligned}
$$

It follows from (A2b) that $y=0$ at $v_{1}=0$ and, consequently, $v_{1}=0$ determines the plane where the potential $v_{0}$ is given. If $v_{1}=1$, then $y=h$ and

$$
x=h\left(u_{1}+A \cdot \operatorname{coth} \frac{\pi u_{1}}{2}\right) .
$$

It is seen that the solution of Eq. (A3) at $u_{1} \geqslant 0$ exists only in the interval of values $x \geqslant x_{m}$ where $x_{m}=x\left(u_{m}\right)$ and $u_{m}$ corresponds to the minimum of the right-hand part of Eq. (A3) and is determined by the ratio

$$
A=\frac{2}{\pi} \sinh ^{2} \frac{\pi u_{m}}{2} .
$$

Analogously, at $u_{1} \leqslant 0$ a solution of Eq. (A3) exists in the interval of values $x \leqslant-x_{m}$. Let us assume $x_{m}=a / 2$, then the constant $A$ can be determined from Eq. (A4). In this case $u_{m}$ is determined by

$$
a=\frac{2}{\pi} h\left(\pi u_{m}+\sinh \pi u_{m}\right) .
$$

Thus, as a result of the conform transformation $z \mapsto w_{1}$ that has been performed the half-plane $y=h, x \geqslant a / 2$ transforms into the area $v_{1}=1, u_{1} \geqslant 0$ where the potential $-v_{h}$ is given, the half-plane $y=h, x \leqslant-a / 2$ transforms into the area $v_{1}$ $=1, u_{1} \leqslant 0$ with the potential $+v_{h}$, and, finally, the plane $y=0$ transforms into the area $v_{1}=0$ with the potential $v_{0}$.

Next conform transformation

$$
w_{2}+u_{2}+i v_{2}=\exp \left(\pi w_{1}\right)
$$

translates the band $0 \leqslant v_{1} \leqslant 1$ onto the real axis $v_{2}=0$ in such a manner that on the real axis of the plane $w_{2}$ the potential is given by the function

$$
U_{a}\left(u_{2}\right)= \begin{cases}-v_{0}, & u_{2} \leqslant-1, \\ +v_{0}, & -1 \leqslant u_{2} \leqslant 0, \\ v_{h}, & u_{2} \geqslant 0 .\end{cases}
$$

We are interested in the potential distribution in the plane $z$ within the interval of values $0 \leqslant y \leqslant h$ that corresponds to the interval $0 \leqslant v_{1} \leqslant 1$ in the plane $w_{1}$ and to the interval $v_{2}$ $\geqslant 0$ in the plane $w_{2}$. According to these definitions the solution of the Laplace equation for the upper half-plane $w_{2}$ at a certain potential distribution along the real axis $U\left(u_{2}\right)$ [Eq. (A7)] is given by Poisson's integral

$$
\begin{aligned}
U_{a}\left(w_{2}\right) & =\frac{1}{\pi} \int_{-\infty}^{+\infty} \frac{U_{a}(t) v_{2} d t}{\left(t-u_{2}\right)^{2}+v_{2}^{2}} \\
& =\frac{1}{\pi}\left[\left(v_{h}-v_{0}\right)\left(\frac{\pi}{2}+\arctan \frac{u_{2}}{v_{2}}\right)+2 v_{0} \arctan \frac{1+u_{2}}{v_{2}}\right] .
\end{aligned}
$$

Translation to the coordinates $u_{1}, v_{1}$ can be done with the help of Eq. (A6) and the ratios 


$$
\frac{u_{2}}{v_{2}}=\cot \pi v_{1}, \quad v_{2}=\sin \pi v_{1} \exp \left(\pi u_{1}\right) .
$$

Finally, $u_{1}, v_{1}$ are expressed by $x, y$ using the system of equations (A2).

Thus, the calculation of the function $U_{a}(x, y)$ based on Eqs. (A2), (A8), and (A9) determines the solution of the auxiliary problem connected with searching the distribution of $U_{a}\left(x, y ; v_{0}, v_{h} ; a\right)$.

${ }^{1}$ T. J. Thornton, M. Pepper, H. Ahmed, D. Andrews, and G. J. Davies, Phys. Rev. Lett. 56, 1198 (1986).
${ }^{2}$ K.-F. Berggren and D. J. Newson, Semicond. Sci. Technol. 1, 327 (1986).

${ }^{3}$ P. L. McEuen, E. B. Foxman, U. Meirav, M. A. Kastner, Y. Meir, and N. S. Wingreen, Phys. Rev. Lett. 66, 1926 (1991).

${ }^{4}$ M. W. Dellow, P. H. Beton, C. J. G. M. Langerak, T. J. Foster, P. C. Main, L. Eaves, M. Henini, S. P. Beaumont, and C. D. W. Wilkinson, Phys. Rev. Lett. 68, 1754 (1992).

${ }^{5}$ Chang-Da Tsai, Ching-Hung Fu, Yow-Jou Lin, and Ching-Ting Lee, Solid-State Electron. 43, 665 (1999).

${ }^{6}$ W. Hansen, A. Schmeller, H. Drexler, J. P. Kotthaus, M. Holland, G. Tränkle, G. Böhm, and G. Weimann, Semicond. Sci. Technol. 9, 1946 (1994).

${ }^{7}$ W. R. Smythe, Static and Dynamic Electricity (McGraw-Hill, New York, Toronto, London, 1950), p. 604. 